\title{
Precoding Aided Data Correlation Scheme for Channel Estimation Technique in MIMO-OFDM System
}

\author{
SIDRAMAYYA S MATAD ${ }^{1}$, RAMESHA K $^{2}$. \\ ${ }^{1}$ Assistant Professor, Department of Electronics \& Communication Engg \\ S. G. Balekundri Institute of Technology \\ Belagavi, VTU Research Scholar Karanataka (State), INDIA \\ ${ }^{2}$ Professor, Department of Electronics \& Communication Engg, Dr.Ambedkar Institute of \\ Technology \\ Bangalore, VTU Research Supervisor, Karanataka (State), INDIA
}

\begin{abstract}
Channel estimation is considered as an important phase in Multiple Input Multiple Output Orthogonal Frequency Division Multiplexing (MIMO-OFDM) networks which can enhances the performance significantly. Channel estimation widely classified as pilot based, blind and semi-blind channel estimation. The pilot-based channel estimation decreases the data transmission rate and spectral efficiency. To overcome these issues of existing schemes, we present a novel blind channel estimation technique. According to proposed scheme, we transmit the data in a block-wise manner. The proposed scheme uses precoding technique to establish the correlation between these blocks. Further, we use channel correlation to solve the diagonal uncertainty of correlation matrix which helps to improve the system performance. We present a comparative analysis study which shows that proposed approach can achieve better performance in terms of Normalized Mean Square Error (NMSE) and Mean Square Error (MSE) when compared with existing techniques.
\end{abstract}

Keywords: Systems Theory, Multiple Input Multiple Output - Orthogonal Frequency Division Multiplexing (MIMO-OFDM), Normalized Mean Square Error, Systems Engineering

Received: October 11, 2020. Revised: February 5, 2021. Accepted: February 18, 2021. Published: March 6, 2021.

\section{Introduction}

Orthogonal Frequency Division Multiplexing (OFDM) system is immensely endorsed in various wireless communication standards like WiMAX, IEEE 802.16, and Long-Term Evaluation (LTE). The main reason behind OFDM's popularity is that it divides wideband into smaller sub bands which are used to convert the frequency selective channel into flat fading. The OFDM technique is preferred for Digital Audio Broadcast (DAB) [1], Digital Video Broadcasting (DVB) [2] and 5 GHz broadband

networks (HIPERLAN/2 and IEEE802.11a) [3]. Moreover, this technique is also embraced for both uplink and downlink communication in 5G systems. Similarly, Multiple-Input Multiple-Output (MIMO) is also deliberated as a favorable technique for $4 \mathrm{G}$ and $5 \mathrm{G}$ wireless communication systems [4]. Zreikat et al. [24] evaluated the performance of $5 \mathrm{G}$ systems where the cell is divided into two zones to represent the WiFI-6 and $5 \mathrm{G}$ technology.

More spectrum efficiency and efficient multipath fading resistance capability are available for the OFDM systems. The cyclic prefix is used to decrease the inter-symbol (ISI) and inter-carrier (ICI) interference in this. Similarly, OFDM utilizes multipath fading channel time delay characteristics to simplify the configuration of the equalizer. 
However, the OFDM and MIMO technologies can be combined to increase the channel capability [5]. In OFDM systems, the wireless channel plays an important role, but it suffers from various difficult problems, such as when information is passed through the wireless fading channel, the signal is corrupted due to Additive White Gaussian Noise (AWGN), which affects the output of the system. Similarly, employing many antennas at the base station can improve the spectral efficiency performance when compared to the current multiantenna LTE systems. However, this gain can be ensued if the Base Station (BS) has the accurate knowledge of Channel State Information (CSI) [6]. Moreover, CSI plays important role for signal detection, channel allocation, resource allocation, beam forming and signal detection. Thus, channel estimation is considered as important phase in MIMO-OFDM systems. Below given Figure 1 shows the general process of channel estimation

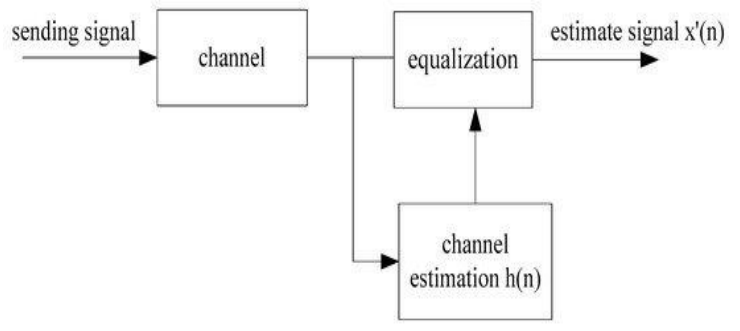

Fig.1: Channel Estimation Procedure

\subsection{Channel Estimation Techniques}

For channel estimation in MIMO-OFDM systems, various techniques have been presented. These techniques can be divided into three major groups, such as preparation, blind and semi-blind channel estimation, or pilot-based approaches.

The pilot tones are inserted into each OFDM symbol or can be inserted into all subcarriers with a particular timeline according to the pilot-based methods. This method of pilot insertion is further known as the pilot insertion process of block type and comb type. Block-type pilot channel estimation: under the slow fading channel, the pilot insertion is assumed in this mechanism. The channel estimation is based on Least Square MMSE for this type of pilot arrangement. When the channel is changing in each OFDM bock, the comb-type pilot channel estimation is introduced for equalization. Such techniques use methods of interpolation. The techniques of blind channel estimation are focused on the exploitation of statistical knowledge from symbols obtained. In terms of saving bandwidth, this scheme is useful.

\section{Literature survey}

This section presents the literature review study about recent techniques of channel estimation in MIMO-OFDM systems. Ma et al. [16] developed channel estimation technique for multi-antenna system for high-mobility scenarios. This work abstracts the channel responses into three domains to report the doubly selective property. The OFDM systems uses time and frequency domains pilots. Initially, time domain training is analyzed to obtain the common support for channel. Later, genetic algorithm-based optimization is implemented for pilot location optimization which helps to construct the compressive sensing structure and recovers the channel. A novel adaptive compressing sensing algorithm is also presented. In [17] Uwaechia et al. considered doubly selective channel and presented a new technique for channel estimation, pilot power allocation and pilot placement. Initially, it exploits the spatial structure of attained response vectors and scarcity of multipath signal components, thus, developed a modified spatial basis expansion model. The complex exponential and modified Complex Exponential Spatial Basis Exponential Model (CESBEM) can be used to represent the downlink communication. Moreover, a joint pilot power and pilot placement strategy is presented for channel estimation.

Mishra et al. [18] presented sparse Bayesian learning scheme for channel estimation in an Orthogonal - STBC, MIMO- OFDM wireless system. A combination of maximum likelihood symbol detection is incorporated in expectation maximization (EM) for ill-posed MIMO-OFDM system. Thus, this scheme performs jointly channel estimation and symbol detection. Some relevant studies about $5 \mathrm{G}$ and wireless communication can be found in [24] and [25]. Hayder et al. [19] 
focused on the issue of pilot contamination proposed a novel approach for channel estimation for MIMO systems. This scheme is based on the sparse Bayesian learning and pattern coupled Gaussian framework. Jeya et al. [20] proposed Optimized Semi-Blind Sparse channel estimation for MIMOOFDM systems. In this approach, Quadrature Phase Shift Keying (QPSK) modulation and Pulse shaping algorithms are implemented at the receiver end for modulation and mitigating the ISI (Inter-Symbol Interferences), respectively. Hansen et al. [21] designed a novel receiver architecture to exchange the information channel estimation and decoding stages. In this receiver, the sparsity based parametric channel estimation is incorporated. The conventional methods restrict the multipath delays however this approach avoids this and reduces leakage effect.

Qin et al. [22] considered time varying channels and presented sparse channel estimation technique for massive MIMO-OFDM systems. Generally, channel estimation of downlink data transmission in MIMO-OFDM is a tedious task because of numerous channel coefficient estimation. AbdzadehZiabari et al. [23] presented a preamble-aided scheme for joint estimation which is used to jointly estimate the time, carrier frequency offset and channel for OFDM systems which are operated in high mobility scenarios. Moreover, this scheme uses Basic Expansion Modeling (BEM) to analyze the time variations which helps to reduce the unknown channel parameters. The BEM coefficients, timing and frequency offset parameters are obtained through the maximum likelihood approach. Below given table 1 presents a comparative analysis of these schemes.

Table.1. Comparative analysis of existing schemes

\begin{tabular}{|c|c|c|}
\hline Reference & Technique & Objective \\
\hline$[16]$ & $\begin{array}{c}\text { Genetic } \\
\text { algorithm }\end{array}$ & $\begin{array}{c}\text { Channel estimation optimization } \\
\text { using genetic algorithm for high- } \\
\text { mobility networks }\end{array}$ \\
\hline$[17]$ & $\begin{array}{c}\text { Spatial Basis } \\
\text { Exponential } \\
\text { Model }\end{array}$ & $\begin{array}{c}\text { Main objective is to improve } \\
\text { channel estimation, pilot power } \\
\text { allocation and pilot placement for } \\
\text { downlink communication. }\end{array}$ \\
\hline$[18]$ & $\begin{array}{c}\text { Bayesian } \\
\text { learning }\end{array}$ & $\begin{array}{c}\text { A joint channel estimation and } \\
\text { symbol detection scheme using } \\
\text { maximum likelihood and } \\
\text { expectation maximization }\end{array}$ \\
\hline
\end{tabular}

\begin{tabular}{|c|c|c|}
\hline$[19]$ & $\begin{array}{c}\text { Bayesian } \\
\text { learning }\end{array}$ & $\begin{array}{c}\text { Channel estimation with } \\
\text { Bayesian learning }\end{array}$ \\
\hline$[20]$ & $\begin{array}{c}\text { QPSK } \\
\text { modulation } \\
\text { and Pulse } \\
\text { shaping } \\
\text { algorithms }\end{array}$ & $\begin{array}{c}\text { The interference cancellation } \\
\text { schemes are combined to perform } \\
\text { the semi-blind channel estimation }\end{array}$ \\
\hline$[21]$ & $\begin{array}{c}\text { Sparsity } \\
\text { based module }\end{array}$ & $\begin{array}{c}\text { New receiver architecture is } \\
\text { designed for channel estimation }\end{array}$ \\
\hline$[23$ & $\begin{array}{c}\text { Basic } \\
\text { Expansion } \\
\text { Modeling }\end{array}$ & $\begin{array}{c}\text { jointly estimate the time, carrier } \\
\text { frequency offset and channel }\end{array}$ \\
\hline \multicolumn{2}{|c}{} \\
\hline
\end{tabular}

\section{Proposed Model}

This section describes the proposed solution for channel estimation in MIMO-OFDM systems. First of all, we present a MIMO-OFDM system model multiple number of transmitter and receiver antennas using different carriers. Later, a channel estimation solution is presented to improve the estimation accuracy. Let us consider a MIMO-OFDM system which is equipped with $N$ number of transmitter antennas and $M$ number of receiver antennas with $K$ number of subcarriers. During transmission, the information bits $b_{i}$, coded bits $c_{i}$ and symbol for $i^{t h}$ stream are transmitted on the $i^{\text {th }}$ antenna on $k^{\text {th }}$ subcarrier. These transmitted symbols are combined and arranged in a vector as $x(k)=$ $\left[x_{1}(k), \ldots . x_{N}(k)\right]^{T}$.

The communication channel between each transmitter and receiver antenna is demonstrated using a conventional digital tapped delay line which contains $L$ taps which are arranged at the OFDM symbol rate. Here, channel coefficient is the important parameter which is used to extract the channel information. The channel coefficient between specific $n^{\text {th }}$ transmitter and $m^{\text {th }}$ receiver is denoted as $L \times 1$ in the vector form as $\boldsymbol{h}_{m n}=$ $\left[h_{m n}(0), h_{m n}(1), \ldots, h_{m n}(L-1)\right]^{T}$. Thus, the collection of channel coefficients for each pair of transmitter and receiver is expressed as:

$$
h=\left[\left[h_{11}^{T}, \ldots . h_{M 1}^{T}\right], \ldots . .,\left[h_{1 N}^{T}, \ldots . h_{M N}^{T}\right]\right]
$$

Here, we assume that channel coefficient $h_{m n}(l)$ for all, $\mathrm{m}, \mathrm{n}$ and $l=0, \ldots L-1$ are independent. Further, we apply Fourier Transform to obtain the channel coefficients in frequency domain for $K$ 
subcarrier. We present a DFT matrix $D$ as $K \times L$ where $k$ and $l^{t h}$ element is denoted as $d_{k l}=e^{-\frac{j 2 \pi k l}{K}}$. Thus, the frequency domain channel coefficients are given as:

$$
\tilde{h}_{m n}(k)=\sum_{l=0}^{L-1} h_{m n}(l) d_{k l}
$$

for all $n=1, \ldots . N, \quad m=1, \ldots M$ and $k=$ $0, \ldots K-1$. The channel matrix for $M \times N$ MIMO system for $k^{\text {th }}$ subcarrier is given as

$$
\widetilde{H}(k)=\left[\begin{array}{cccc}
\tilde{h}_{11}(k) & \tilde{h}_{12}(k) & \ldots & \tilde{h}_{1 N}(k) \\
\tilde{h}_{21}(k) & \tilde{h}_{22}(k) & \ldots & \tilde{h}_{2 N}(k) \\
\vdots & \vdots & \vdots & \vdots \\
\tilde{h}_{M 1}(k) & \ldots & \ldots & \tilde{h}_{M N}(k)
\end{array}\right]
$$

With the help of this, the $M \times 1$ received signal vector for $k^{\text {th }}$ subcarrier is given by:

$$
y(k)=\widetilde{\boldsymbol{H}}(k) x(k)+w(k) \quad \forall k=0,1, \ldots K-1
$$

Where $w(k)$ is a noise vector of size $M \times 1$ which is a symmetric complex Gaussian random variable. Each antenna contributes some noise in the received signal. The noise variance for each antenna is denoted by $\gamma^{-1}$. Similarly, the scalar form of the received signal is given as:

$$
y_{m}(k)=\sum_{n=1}^{N} x_{n}(k) \sum_{l=0}^{L-1} h_{m n}(l) d_{k l}+w_{m}(k)
$$

Where $y_{m}$ and $w_{m}$ represent the $m^{\text {th }}$ element of $y(k)$ and $w(k)$ respectively. Final received signal and channel matrix are given as

$$
\begin{array}{r}
y=\left[y(0)^{T}, \ldots y(K-1)^{T}\right]^{T} \\
\widetilde{H}=\left[\widetilde{H}(0)^{T}, \ldots \widetilde{H}(K-1)^{T}\right]^{T}
\end{array}
$$

In this work, our aim is to estimate the accurate value of channel matrix and its coefficients to improve the performance of MIMO-OFDM system.

In order to achieve this, let us consider that $i^{\text {th }}$ block of symbol of $p^{\text {th }}$ user is given as $d_{p}^{i} \triangleq$ $\left[d_{p}^{i}(0) \ldots d_{p}^{i}(N-1)\right]^{T}$ without considering any precoding. In this $i^{\text {th }}$ block, the symbol of $p^{\text {th }}$ user are transmitted over carrier $k$. These symbols are generated in a vector form as $N \times 1$ as a code vector which can be expressed as:

$$
s_{p}^{i}(k) \triangleq w_{p}^{H}(k ; i) d_{p}^{i}
$$

Similarly, we perform thee precoding on $i^{\text {th }}$ block of $p^{\text {th }}$ user which is given as:

$$
\begin{gathered}
s_{p}^{i}(k) \triangleq\left[s_{p}^{i}(0), \ldots . s_{p}^{i}(N-1)\right]^{T} \\
=\left[w_{p}(0 ; i), w_{p}(1 ; i), \ldots ., w_{p}(N-1 ; i)\right]^{H} d_{p}^{i} \\
=W_{p}^{i} d_{p}^{i}
\end{gathered}
$$

During the transmission of these data blocks, the noise deteriorates the quality and also signals suffer from the interference. Hence, the main of this precoding is to introduce correlation between transmitted blocks. Moreover, a diversity also can be introduced which helps to estimate the channel matrix. This diversity is incorporated by changing the coding matrix.

On the other hand, let us consider that $i^{\text {th }}$ block is received by the receiver antenna. The symbol of all users on carrier $k$ are arranged in a vector form $y^{i}(k)$. This can be expressed as:

$$
\begin{gathered}
y^{i}(k) \triangleq\left[y_{o}^{i}(k) \ldots y_{M R-1}^{i}(k)\right]^{T}=H(k) s^{i}(k)+ \\
n(k)(9)
\end{gathered}
$$

Where $s^{i}(k) \triangleq\left[s_{0}^{i}(k) \ldots s_{M_{T-1}}^{i}(k)\right]^{T}$. With help of Eq. (7) and (9), the received signal vector can be rewritten as:

$$
y^{i}(k)=H(k) \psi^{i}(k) d^{i}+n(k)
$$

Where

$$
\psi^{i}(k) \triangleq\left[\begin{array}{cccc}
w_{0}^{H}(k ; i) & 0 & \ldots & 0 \\
0 & w_{1}^{H}(k ; i) & \ldots & 0 \\
\vdots & \vdots & \vdots & \vdots \\
0 & 0 & \ldots & w_{M_{T-1}^{H}}^{H}(k ; i)
\end{array}\right]
$$

$$
\text { And } d^{i} \triangleq\left[\left(d_{0}^{i}\right)^{T},\left(d_{1}^{i}\right)^{T}, \ldots\left(d_{M}^{i}\right)^{T}\right]^{T}
$$

In general, estimating $\boldsymbol{H}(k)$ for fixed $k$ is a common MIMO channel estimation problem. However, we 
focus on varying $k$ and estimating the channels for received blocks. Let $\tilde{y}^{i}$ denotes the received symbol sequence which starts at block $i$ and spaced apart by $M$ blocks. This sequence is expressed as:

$$
\tilde{y}^{i}(k)=\left\{y^{i}(k), y^{i+M}(k), y^{i+2 M}(k), \ldots\right\}
$$

The correlation matrix of received sequence is presented as:

$$
R_{k l}^{i}=\left\{y^{i}(k), y^{i+M}(k), y^{i+2 M}(k), \ldots\right\}
$$

With the help of Eq. (12), the correlation can be rewritten as:

$$
\begin{array}{r}
R_{k l}^{i}=\sigma^{2} H(k) \operatorname{Diag}\left(w_{k l}^{i}\right) H^{H}(l)+\delta_{k, l} \sigma_{n}^{2} I_{M_{r}}, \\
i=0, \ldots M-1
\end{array}
$$

In order to extract the $u^{\text {th }}$ column of $R_{k l}^{i}$, we multiply $R_{k l}^{i}$ with column selection vector $e_{u}$. Based on Eq. (13), it can be given as:

$$
\begin{gathered}
R_{k l}^{i} e_{u}=\sigma^{2} H(k) \operatorname{Diag}\left[H^{H}(l) e_{u}\right] w_{k l}^{i} \\
+\delta_{k, l} \sigma_{n}^{2} e_{u}
\end{gathered}
$$

Thus the matrix $C_{u k l}$ can be constructed based on the $u^{t h}$ column of matrices $R_{k l}^{0}, R_{k l}^{1}, \ldots, R_{k l}^{M_{T}-1}$. This is expressed as:

$$
\begin{gathered}
C_{u k l}=\frac{1}{\sigma^{2}}\left[R_{k l}^{0} e_{u}, R_{k l}^{1} e_{u}, \ldots, R_{k l}^{M_{T}-1} e_{u}\right] \\
=H(k) \operatorname{Diag}\left[H^{H}(l) e_{u}\right] U_{k l}+\delta_{k l} \frac{\sigma_{n}^{2}}{\sigma^{2}} e_{u}
\end{gathered}
$$

At this phase, we can transmit the pilots using $l^{\text {th }}$ carrier based on which we estimate the value of $H(l)$. However, we precoding is applied then it is not feasible to transmit pilots hence we will construct a set of indices as $\left\{p_{1}, p_{2}, p_{3}, \ldots p_{K}\right\}$ which will not be precoded. Based on these blocks the estimation can be presented as: $\widehat{\boldsymbol{H}}(l) \approx$ $\left[y^{p_{1}}(l), y^{p_{2}}(l), \ldots y^{p_{K}}(l)\right]\left[s^{p_{1}}(l), s^{p_{2}}(l), \ldots s^{p_{K}}(l)\right]$

\section{Results and Discussion}

In this section, we discussed the experimental analysis using proposed method and investigated and compared the outcome of proposed scheme with various existing techniques. The complete experimental study is performed using MATLAB simulation tool on PC which has Intel core i5 CPU, 3.2 GHZ with 16GB memory and $8 \mathrm{~GB}$ of GPU memory. The simulation parameters considered in this work are presented in below given table 2 .

Table.2. Simulation Parameter

\begin{tabular}{|c|c|}
\hline $\begin{array}{c}\text { Simulation Parameter } \\
\text { Name }\end{array}$ & Used Parameter value \\
\hline $\begin{array}{c}\text { Number of transmitter } \\
\text { antennas }\end{array}$ & 64 \\
\hline $\begin{array}{c}\text { Number of receiver } \\
\text { antennas }\end{array}$ & 64 \\
\hline Modulation & QPSK \\
\hline Number of subcarriers & 2048 \\
\hline Length of cyclic prefix & 256 \\
\hline Number of taps & 90 \\
\hline
\end{tabular}

We consider a MIMO-OFDM system with 64 transmitter and receiver antennas. The data transmission is performed using QPSK modulation system with the help of 2048 number of subcarriers. This system uses 256 as a cyclic prefix and 90 as the number of taps. The performance of system is calculated in terms of MSE, NMSE and BER. The signal to noise ratio per transmitter antenna is expressed as

$$
S N R=\frac{\sigma_{h}^{2}(L+1)}{\sigma_{e}^{2}}
$$

Where $L$ denotes the fading, $\sigma_{h}^{2}$ is the variance of the channel taps and $\sigma_{e}^{2}$ denotes the noise variance. Similarly, we compute the NMSE as follows:

$$
N M S E=\frac{1}{R} \sum_{r=0}^{R-1} \frac{\left\|\hat{h}^{(r)}-h^{(r)}\right\|^{2}}{(L+1) N_{T} N_{R} \sigma_{h}^{2}}
$$

where $R$ represents the total number of simulations runs, $N_{T}$ represents the number of transmitter and $N_{R}$ denotes the number of receivers

Further, we compare the efficiency of proposed method with existing techniques for varied number of pilots and SNRs. First, we consider fixed pilot numbers as 8 and 16 with varied SNR. Later, we have varied the number of pilots and fixed the SNR 
as $15 \mathrm{~dB}$ to present the comparative analysis. Below given figure 2 shows a comparative study in terms of MSE for varied SNR. The obtained outcome is compared with Deep low-SNR [18], Deep high-SNR [18], and CAE-Channel Net [18].

Figure 2 shows that proposed approach achieves better performance by reducing the mean squared error at the receiver end. Initially, the low SNR values produce more error due to less correlation between the data. As the SNR is increasing, error also reduces.

The existing scheme seems to obtain the saturated point for MSE reduction at the $20 \mathrm{~dB}$ of SNR level whereas proposed approach further improves the performance. This study shows that the average MSE is obtained as $0.0753,0.093,0.1778$ and 0.2084 using proposed approach, CAE channel net, Deep high SNR and Deep Low SNR respectively.

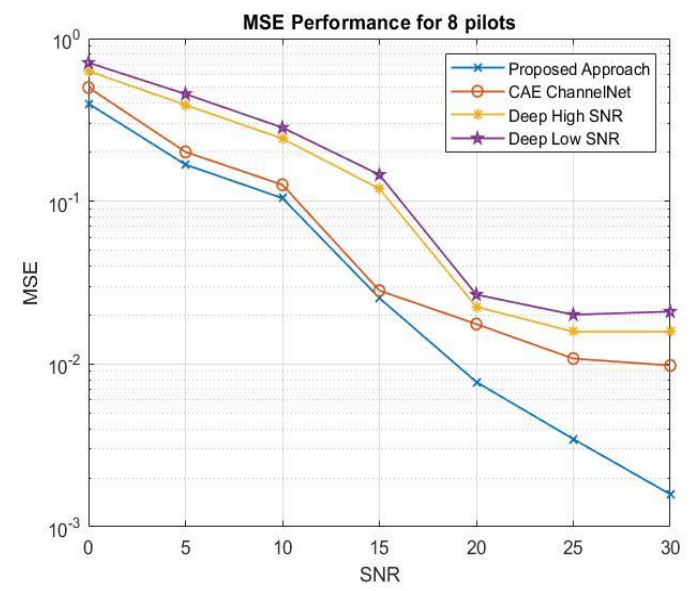

Fig.2: MSE Performance for 8 Pilot Scenario

In next experiment, we perform the experiment for 16 number of pilots for varied number of SNRs. Below given Figure 3 shows the outcome of this experiment

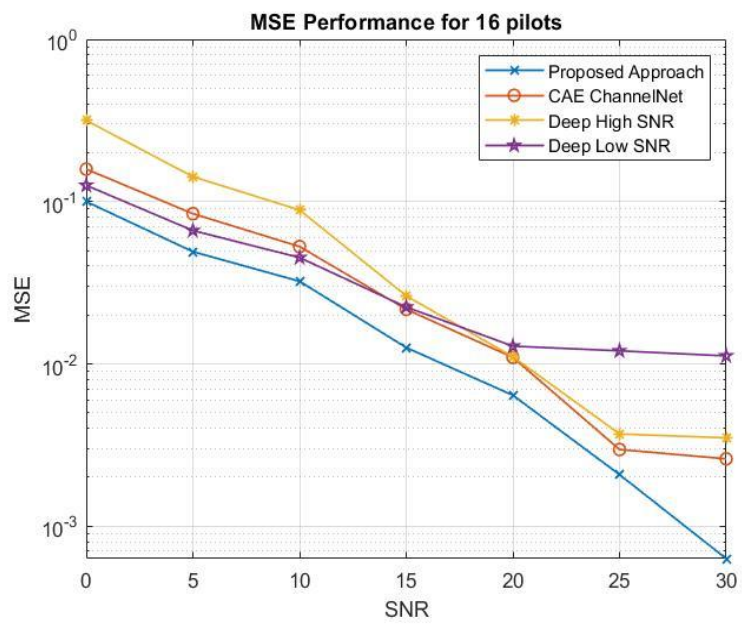

Fig.3: MSE Performance for 16 Pilots

In this experiment we obtain the average the average performance as $0.0234,0.0356,0.0385$ and 0.0644 by using proposed approach, Deep Low SNR, CAE Channel Net and Deep High SNR, respectively. The existing scheme are not suitable to achieve the high SNR values because after achieving the certain SNR, it doesn't improve further.

In next experiment, we fix the SNR at $15 \mathrm{~dB}$ and varied the number of pilots from 10 to 50 . The obtained performance is depicted and compared in below given 4 Figure

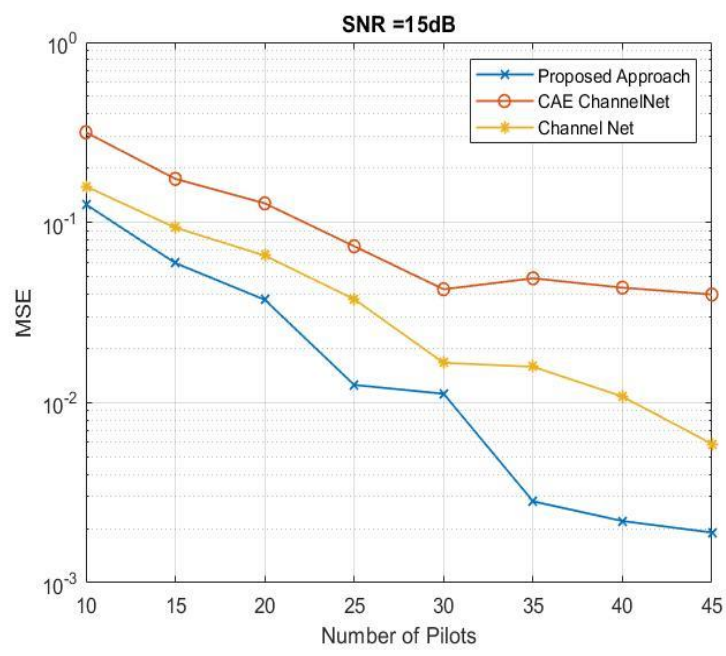

Fig.4.: MSE performance for varied pilots

According to this experiment, we obtain that the proposed approach achieves better performance as number of pilots are increasing. The experiment shows that MSE of Channel Net and CAE-Channel 
Net follows the uniform for convergence whereas proposed approach maintains the reliable performance. Hence, selection of number of pilots is an important task when low number of pilots are used. Similarly, we measure the performance in terms of NMSE and compare with existing techniques such as DF method [19], LS method [19], and Haar wavelet method [19]. Below given Figure 5 shows the comparative performance for varied SNR. This comparative analysis shows that proposed approach meets better performance when compared with conventional techniques. The average NMSE is obtained as 0.2778, 0.0521, 0.1107 and 0.0320 using DF, Haar, LS and proposed approach respectively. This comparison shows that performance of proposed approach is improved by $88.48 \%, 81.25 \%$, and $60.15 \%$ when compared with DF, Haar, and LS algorithms. The proposed precoding and correlation techniques helps to minimize the symbol contamination and improves the channel estimation which mitigates the performance related issues of proposed approach.

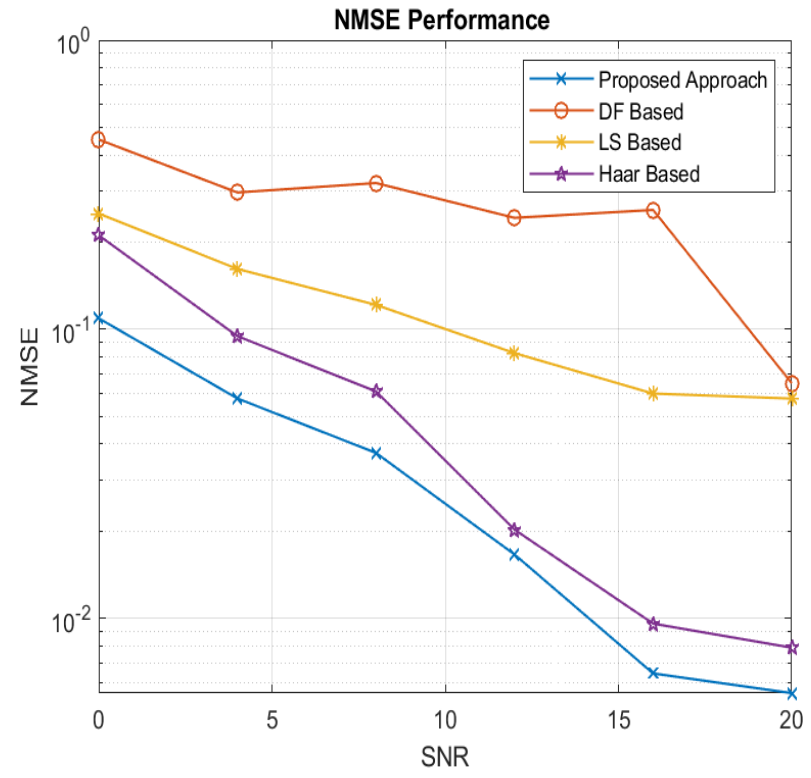

Fig.5: NMSE Performance

\section{Conclusion}

Demand of wireless cellular communication is increasing rapidly hence providing efficient resources and maintaining network in such a way that it can meet requirements, is a tedious task. Currently, MIMO-OFDM technique has gained more attraction from research community and industries due to their vital role in cellular communication. However, channel estimation is an ambitious task in these systems which degrades the system performance. In this work, we focused on channel estimation and presented a novel approach. The proposed approach uses precoding to establish the correlation between transmitted blocks. Further, a novel mechanism is described to obtain the channel coefficients. These coefficients suffer from diagonal uncertainty issues hence precoding correlation is used to solve this problem. Experimental results shows that proposed approach achieves the better performance with respect to MSE and NMSE. In future, this work can be extended by incorporating deep learning schemes for channel estimation and interference cancellation for massive MIMO systems.

\section{Acknowledgment}

Authors would like to sincerely thank Dr. Nanjundaswamy C, Principal, Dr.Ambedkar Institute of Technology ,Bangalore, Karnataka. and Dr. Sidramappa V.Itti, Principal, S. G. Balekundri Institute of Technology, Belagavi, Karnataka and dear staff member of Dr.AIT, Bangalore and SGBIT, Belagavi for their kind support in carrying out this research work.

\section{References:}

[1]. Sim, Zee Ang, Regina Reine, Zhuquan Zang, and Lenin Gopal. "PAPR and BER reduction in MU-MIMO-OFDM systems via a set of waveforms." In 2017 IEEE International Conference on Signal and Image Processing Applications (ICSIPA), pp. 55-60. IEEE, 2017.

[2]. Acar, Yusuf, Sultan ALDIRMAZ Çolak, and Ertugrul Basar. "Channel estimation for OFDM-IM systems." Turkish Journal of Electrical Engineering \& Computer Sciences 27, no. 3 (2019): 1908. 
[3]. Wang, Jiaming, and Chao Chen. "Novel Digital Audio Broadcasting System Based on CE-OFDM and FM." In 2018 International Conference on Information Systems and Computer Aided Education (ICISCAE), pp. 46-50. IEEE, 2018.

[4]. Elijah, Olakunle, Chee Yen Leow, Tharek Abdul Rahman, Solomon Nunoo, and Solomon Zakwoi Iliya. "A comprehensive survey of pilot contamination in massive MIMO-5G system." IEEE Communications Surveys \& Tutorials 18, no. 2 (2015): 905-923.

[5]. Liu, Yongjun, Guisheng Liao, Zhiwei Yang, and Jingwei $\mathrm{Xu}$. "Design of integrated radar and communication system based on MIMO-OFDM waveform." Journal of Systems Engineering and Electronics 28, no. 4 (2017): 669-680.

[6]. Björnson, Emil, Luca Sanguinetti, Henk Wymeersch, Jakob Hoydis, and Thomas L. Marzetta. "Massive MIMO is a realityWhat is next?: Five promising research directions for antenna arrays." Digital Signal Processing 94 (2019): 3-20.

[7]. Dong, Peihao, Hua Zhang, Geoffrey Ye Li, Ivan Simões Gaspar, and Navid NaderiAlizadeh. "Deep CNN-based channel estimation for mmWave massive MIMO systems." IEEE Journal of Selected Topics in Signal Processing 13, no. 5 (2019): 9891000 .

[8]. Yang, Yuwen, Feifei Gao, Xiaoli Ma, and Shun Zhang. "Deep learning-based channel estimation for doubly selective fading channels." IEEE Access 7 (2019): 3657936589.

[9]. Nandi, Shovon, Narendra Nath Pathak, and Arnab Nandi. "A Novel Adaptive Optimized Fast Blind Channel Estimation for Cyclic Prefix Assisted Space-Time Block Coded MIMO-OFDM Systems." Wireless Personal Communications 115, no. 2 (2020): 13171333.

[10]. Liu, Yu, and Fanggang Wang. "Blind Channel Estimation and Data Detection with Unknown Modulation and
Coding Scheme." arXiv preprint arXiv:1909.11306 (2019).

[11]. Wang, Han, Jianqing Liao, Lingwei $\mathrm{Xu}$, and Xianpeng Wang. "Blind channel estimation for FBMC/OQAM systems based on subspace approach." Information 9, no. 3 (2018): 58.

[12]. Shen, Lei, Yu-Dong Yao, Haiquan Wang, and Huaxia Wang. "Blind decoding based on independent component analysis for a massive MIMO uplink system in microcell Rician/Rayleigh fading channels." IEEE Transactions on Vehicular Technology 65, no. 10 (2015): 8322-8330.

[13]. Pasangi, Parisa, Mahmoud Atashbar, and Mahmood Mohassel Feghhi. "Blind downlink channel estimation of multi-user multi-cell massive MIMO system in presence of the pilot contamination." AEU-International Journal of Electronics and Communications 117 (2020): 153099.

[14]. $\mathrm{Hu}$, Cheng, Hong Wang, and Rongfang Song. "Analysis of Semi-Blind Channel Estimation in Multiuser Massive MIMO Systems With Perturbations." IEEE Access 7 (2019): 147872-147882.

[15]. Liu, Changjiang, and Tughrul Arslan. "RecNet: Deep Learning-Based OFDM Receiver with Semi-Blind Channel Estimation." In 2020 IEEE International Symposium on Circuits and Systems (ISCAS), pp. 1-4. IEEE, 2020.

[16]. Ma, Xu, Fang Yang, Sicong Liu, Jian Song, and Zhu Han. "Sparse channel estimation for MIMO-OFDM systems in high-mobility situations." IEEE Transactions on Vehicular Technology 67, no. 7 (2018): 6113-6124.

[17]. Uwaechia, Anthony Ngozichukwuka, Nor Muzlifah Mahyuddin, Mohd Fadzil Ain, Nurul Muazzah Abdul Latiff, and Nor Farahidah Za'bah. "Compressed channel estimation for massive MIMO-OFDM systems over doubly selective channels." Physical Communication 36 (2019): 100771. 
[18]. Soltani, Mehran, Vahid Pourahmadi, and Hamid Sheikhzadeh. "Pilot Pattern Design for Deep Learning-Based Channel Estimation in OFDM Systems." IEEE Wireless Communications Letters 9, no. 12 (2020): 2173-2176.

[19]. Tang, Ruiguang, Xiao Zhou, and Chengyou Wang. "A Haar wavelet decision feedback channel estimation method in OFDM systems." Applied Sciences 8, no. 6 (2018): 877.

[20]. Jeya, R., \& Amutha, B. (2019). Optimized semiblind sparse channel estimation algorithm for MU-MIMO OFDM system. Computer Communications, 146, 103-109.

[21]. Hansen, T. L., Jørgensen, P. B., Badiu, M. A., \& Fleury, B. H. (2018). An iterative receiver for OFDM with sparsity-based parametric channel estimation. IEEE Transactions on Signal Processing, 66(20), 5454-5469.

[22]. Qin, Q., Gui, L., Gong, B., \& Luo, S. (2018). Sparse channel estimation for massive MIMO-OFDM systems over time-varying channels. IEEE Access, 6, 33740-33751.

[23]. Abdzadeh-Ziabari, H., Zhu, W. P., \& Swamy, M. N. S. (2017). Joint maximum likelihood timing, frequency offset, and doubly selective channel estimation for OFDM systems. IEEE Transactions on Vehicular Technology, 67(3), 2787-2791.

[24]. Aymen Zreikat, Performance Evaluation of 5G/WiFi-6 Coexistence, International Journal of Circuits, Systems and Signal Processing, Volume 14, 2020, pp. 903-913.

[25]. Michael H. Schwarz, Larissa Gaus, Josef Borcsok, Investigation of a Safety Parameter Observer for Wireless Communication, International Journal of Circuits, Systems and Signal Processing, Volume 14, 2020, pp. 1005-1010.

\section{Contribution of individual authors to the creation of a scientific article (ghostwriting policy)}

Sidramayya S M: Has conceived the presented idea, methodology, implementation, writing, reviewing, and editing.

Follow: www.wseas.org/multimedia/contributorrole-instruction.pdf

\section{Creative Commons Attribution \\ License 4.0(Attribution 4.0 \\ International, CC BY 4.0)}

This article is published under the terms of the Creative Commons Attribution License 4.0 https://creativecommons.org/licenses/by/4.0/dee d.en_US

\section{Creative Commons Attribution License 4.0 (Attribution 4.0 International, CC BY 4.0)}

This article is published under the terms of the Creative Commons Attribution License 4.0 https://creativecommons.org/licenses/by/4.0/deed.en US 